\title{
Evaluation of Recovery and Monitoring Methods for Parasitoids Released Against Emerald Ash Borer
}

Michael S. Parisio ${ }^{\text {a, }}$, Juli R. Gould ${ }^{\text {b }}$, John D. Vandenberg ${ }^{\text {c }}$, Leah S. Bauer ${ }^{\mathrm{d}}$, and Melissa K. Fierke $^{\mathrm{a}}$ 
Emerald ash borer (Agrilus planipennis Fairmaire, EAB) is an invasive forest pest and the

16 target of an extensive biological control program designed to mitigate EAB-caused ash (Fraxinus

17 spp.) mortality. Since 2007, hymenopteran parasitoids of EAB from northeastern Asia have been

18 released as biological control agents in North America, including Oobius agrili (Hymenoptera:

19 Encyrtidae), an egg parasitoid; Tetrastichus planipennisi (Hymenoptera: Eulophidae), a larval

20 endoparasitoid; and Spathius agrili (Hymenoptera: Braconidae), a larval ectoparasitoid.

21 Following parasitoid releases in new locations, methods currently used to document presence

22 and establishment and to monitor dispersal of parasitoids in the field were simultaneously

23 evaluated, including destructive sampling of entire trees and deployment of egg sentinel logs

24 (ESLs), egg sentinel cups (ESCs), larval sentinel logs (LSLs), and yellow pan traps (YPTs). All

25 three parasitoids were recovered using YPTs and destructive sampling of trees. Spathius agrili

26 was the only species to be recovered using LSLs, however, results indicate YPTs were as

27 effective as LSLs. YPTs were also as effective as destructive sampling of entire trees for $O$.

28 agrili and S. agrili detection. YPT trap catches were significantly associated with egg parasitism

29 on sampled trees by O. agrili, but not for larval parasitoids. Additional research indicated YPTs

30 are effective in recovering $O$. agrili and $T$. planipennisi at distances as great as $20 \mathrm{~m}$ from release

31 points. It is therefore recommended that YPTs be used as the preferred method for parasitoid

32 recovery as the other methods are much more labor intensive and prone to difficulties. 
36 KEY WORDS: Yellow pan trap, emerald ash borer, biological control, parasitoid, Spathius agrili,

37 Tetrastichus planipennisi, Oobius agrili

38

39

40

41

42

43 


\section{Introduction}

The emerald ash borer (EAB, Agrilus planipennis Fairmaire, Coleoptera: Buprestidae) is an invasive wood-boring beetle responsible for the deaths of hundreds of millions of ash trees

47 (Fraxinus spp.) in North America (Cappaert et al. 2005, Herms and McCullough 2014, emeraldashborer.info 2016). Given the current scale of infestation, management of EAB in forest settings now primarily relies on a biological control program intended to provide long-term population suppression and mitigate future ecological impacts of EAB to the greatest extent

51 possible (Bauer et al. 2015).

52 Exploratory surveys for natural enemies within areas of EAB's native range in

53 northeastern China and the Russian Far East have resulted in the identification of four major

54 hymenopteran parasitoids: Oobius agrili Zhang and Huang (Hymenoptera: Encyrtidae), an egg

55 parasitoid (Zhang et al. 2005); Tetrastichus planipennisi Yang (Hymenoptera: Eulophidae), a

56 larval endoparasitoid (Liu et al. 2003, Yang et al. 2006); and the larval ectoparasitoids Spathius

57 agrili Yang (Yang et al. 2005), and Spathius galinae Belokobylskij \& Strazanac (Hymenoptera:

58 Braconidae) (Belokobylskij et al. 2012). Following research on EAB population dynamics in

59 China (Liu et al. 2007) and host specificity testing (Federal Register 2007, Federal Register

60 2015), these species were approved for release in the United States and are now mass reared for

61 release by USDA (Federal Register 2007, Federal Register 2015, Bauer et al. 2015). Releases of

62 O. agrili, T. planipennisi, and S. agrili began in 2007, and S. galinae releases in 2015.

63 Since 2007, both $T$. planipennisi and $O$. agrili have been recovered in nine states and $S$.

64 agrili in six states (mapbiocontrol 2016), demonstrating the ability of these introduced

65 parasitoids to reproduce in the field and survive at least one winter over a large geographic area.

66 Establishment of biological control agents, defined as sustained populations for at least two years 
67 after final field release in an area, has been confirmed for $O$. agrili and T. planipennisi at long-

68 term study sites in Michigan and other states (Abell et al. 2014, Bauer et al. 2015, Duan et al.

69 2013, 2015). Despite the apparent ability to overwinter in some northern states, S. agrili has not

70 been documented to persist more than two years, possibly due to asynchrony with its host. Long-

71 term monitoring of parasitoids after release is essential to determining successful establishment

72 and evaluating impacts of parasitoids on EAB populations as well as ash survival and

73 regeneration. During initial establishment of parasitoids, small populations make field recovery

74 difficult and so great effort has been invested in developing effective parasitoid detection and

75 monitoring methods.

76 One sampling method to detect presence of EAB parasitoids involves felling EAB-

77 infested ash trees, cutting them into logs, incubating logs in cardboard rearing tubes, and

78 identifying emerging adult parasitoids (Bauer et al. 2011, Abell et al. 2015, Gould et al. 2016).

79 This method isn't conducive for documenting egg parasitism as $O$. agrili is very small, and so an

80 additional step is needed to examine the bark of infested ash trees for EAB eggs, followed by

81 debarking and collecting/dissecting EAB larvae (Abell et al. 2014, Duan et al 2013, 2015). As

82 the EAB biological control program expands in North America, more efficient non-destructive

83 monitoring methods are needed for detection of all EAB biocontrol agents in the field to evaluate

84 their establishment and spread.

85 The use of sentinel eggs or larvae, reared from EAB in the laboratory and placed in the

86 field to attract egg or larval parasitoids, is another method of monitoring parasitoid establishment

87 (Bauer et al. 2011, Duan et al. 2012, Jennings et al. 2014). However, rearing and preparing EAB

88 eggs and larvae for use as sentinels is time-consuming, technically challenging, and restricted to

89 those with the knowledge, facilities, and materials required to successfully rear EAB. 
Colored pan traps are widely used collecting method for insects and have been used

91 previously to survey parasitic hymenopterans (Noyes 1989, Pucci 2008). High-reflectance colors

92 like yellow or white perform well and yellow pan traps (YPTs) have been used successfully to

93 recover EAB parasitoids (Abell et al. 2015, Bauer et al. 2011, 2016, Vrdoljak et al. 2012). This

94 method is relatively inexpensive, requiring purchase and assembly of plastic yellow bowls with

95 zip ties and a shelf bracket, and does not require a laboratory or explicit technical skills.

96 While the aforementioned methods have all been used to recover parasitoids, they were

97 used in independent experiments and to our knowledge no one has carried out side by side field

98 comparisons. Thus, the primary objective of this study was to evaluate these methods

99 simultaneously to determine which are most effective at capturing EAB parasitoids. In addition

100 to comparing these methods, we also used data from destructively sampling trees to determine if

101 any of the methods could be used to indicate parasitism within the trees to which traps were

102 attached.

103 Although recovery of adult larval parasitoids in YPTs has been documented (Bauer et al.

1042011,2016 ), we also wished to confirm a more recent observation (J Gould) that the egg

105 parasitoid, O. agrili, can also be reliably recovered in these traps. Finally, the effective range of

106 YPTs was investigated to determine whether these traps can recover parasitoids at greater

107 distances not immediately adjacent from the original release point.

108

109 2. Materials and Methods

$110 \quad$ 2.1 Comparison of Parasitoid Recovery Methods

$111 \quad 2.1 .1$ Release sites 
112 Two parasitoid release sites $\sim 2 \mathrm{~km}$ apart were selected near Selkirk in Albany County,

113 NY within mid-successional, EAB-infested forest stands $\sim 5$ ha in size and dominated by white

114 ash (Fraxinus americana L.). A stand on Currey Ave (N 42.552229, W -73. 846312) consisted of

$115 \sim 75 \%$ white ash and showed signs of moderate EAB infestation. A stand on West Yard Rd (N

$11642.566105, \mathrm{~W}-73.863208$ ) also consisted of $\sim 75 \%$ white ash, however trees already exhibited

117 signs of a more advanced infestation, including visible symptoms of EAB-induced dieback and 118 some tree mortality.

119 A single cluster of 10 live ash trees with clear signs of EAB infestation, especially

120 woodpecker foraging, was selected at each of the two sites. Trees were girdled in mid May 2013

121 by removing a $15 \mathrm{~cm}$ strip of bark from the trunk at a height of $1 \mathrm{~m}$ to attract EAB emerging in

122 Jun and Jul, thereby concentrating within-tree EAB populations and increasing the number of

123 EAB eggs and larvae available for parasitoids (McCullough et al. 2009). Girdled trees had a

124 mean $\mathrm{DBH}$ of $9.4 \mathrm{~cm}( \pm 0.6 \mathrm{SE}$, range $6.8 \mathrm{~cm}$ to $13.0 \mathrm{~cm})$ at Currey Ave and $11.7 \mathrm{~cm}( \pm 0.5 \mathrm{SE}$,

125 range $7.8 \mathrm{~cm}$ to $13.9 \mathrm{~cm}$ ) at West Yard $\mathrm{Rd}$.

126

127 2.1.2 Parasitoid Releases

128 Oobius agrili were disseminated from release devices placed at a height of $1.5 \mathrm{~m}$ on the

12910 girdled ash trees at both field sites on two occasions (20 total release devices) in Jun/Jul of

130 2013. Oobius agrili were received from the USDA-APHIS-PPQ Biological Control Rearing

131 Facility in Brighton, MI as late-instar larvae or pupae inside parasitized EAB eggs laid on filter

132 paper. Filter paper was placed in a field release device modeled after "Oobinators" (See USDA

133 APHIS/ARS/FS 2016), with minor modifications to structure but not overall function.

134 Approximately 2,400 parasitized EAB eggs were deployed in total. At each site, 600 eggs were 
135 deployed at the beginning of the experiment, coinciding with the beginning of the EAB egg

136 laying season ( 2-3 weeks after adult EAB emergence), and an additional 600 eggs were

137 deployed two weeks later (Currey Ave on 21-Jun and 6-Jul, West Yard Rd on 28-Jun and 12-

138 Jul). Parasitized eggs were distributed evenly among 10 release devices so that each contained

$139 \sim 60$ parasitized eggs and O. agrili completed development within the release devices under

140 natural ambient conditions. To encourage survival, the inside surface of release devices were

141 provisioned with streaks of honey to serve as an initial food and liquid resource for emerging $O$.

142 agrili adults (L Bauer). Six weeks after release, filter paper was collected and eggs examined

143 under a dissecting microscope for $O$. agrili exit holes to quantify the actual number of adult

144 parasitoids to successfully emerge.

145 Spathius agrili and T. planipennisi were both disseminated weekly from 10 emergence

$146 \log$ at each site over a four week period from Aug through early Sept 2013 (note that this time

147 period did not overlap with release of $O$. agrili). Larval parasitoids were received from the

148 Brighton USDA-APHIS-PPQ Biological Control Rearing Facility as late-instar larvae or pupae

149 inside parasitized EAB larvae in small ash bolts $\sim 15 \mathrm{~cm}$ long and $\sim 5.5 \mathrm{~cm}$ in diameter. They

150 were attached to non-girdled ash trees dispersed throughout the two release sites. Larval

151 parasitoids completed development and emerged as adults under these natural conditions. A new

152 set of emergence logs was received and placed at both sites weekly for four consecutive weeks.

153 The goal was to release sufficient numbers of both larval parasitoids and preliminary estimates

154 from the Brighton lab indicated $\sim 1,200$ S. agrili and 3,340 T. planipennisi were expected to

155 emerge from bolts deployed at each site. This number was recalculated once all parasitoids had

156 emerged by removing the outer bark of emergence logs to count individual S. agrili cocoons with

157 exit holes and estimate T. planipennisi numbers based on the actual number of EAB larvae 
158 parasitized and the mean brood size and number of broods per log. Mean brood size was

159 generated using fully-developed broods of $T$. plannipennisi adults that failed to emerge $(\mathrm{n}=68)$

160 from $\operatorname{logs}($ mean $=32$ per parasitized larvae $)$.

161

\section{2.1.3 Parasitoid Recoveries}

163 Egg sentinel logs (ESLs) used for $O$. agrili recovery were prepared using methods

164 adapted from those reported in Duan et al. (2012) using small white ash logs $(\sim 15 \mathrm{~cm}$ long, $\sim 5.5$

$165 \mathrm{~cm}$ in diameter) with freshly laid EAB eggs on the bark surface. Adult female EAB for egg

166 production were collected from local ash logs placed in rearing tubes with emerging adults being

167 collected every $1-2$ days and transferred to plastic rearing containers $(12 \times 12 \times 15 \mathrm{~cm})$. To

168 facilitate EAB mating, 1-2 males and 3-5 females were kept in each container and dead adults

169 were replaced as needed to maintain these relative numbers. Each ESL was wrapped in $5 \mathrm{~mm}$

170 wide purple curling ribbon to create concealed oviposition sites preferred by EAB females and

171 placed in a container with mated females for 3-5 days prior to placement in the field. Eggs laid

172 beneath the ribbon were counted and circled with fine-point permanent marker prior to being

173 placed in the field. Only 16 ESLs were produced (due to difficulties encountered), with egg

174 densities ranging from 3-21 EAB eggs per $\log (\mathrm{n}=140$ eggs total). Oobius agrili prefers freshly

175 laid EAB eggs (Duan et al. 2014), therefore we made certain that EAB eggs were <5 days old at 176 the time they were placed in the field. Eggs on ESLs were exposed to parasitism for a one week

177 period and ESLs were replaced weekly for four weeks from late Jun through mid Jul 2013. Due

178 to low ESL availability, all ESLs produced in a given week (3-5) were placed on girdled trees at 179 a single site. Sites were alternated weekly rather than distributing limited ESLs between both 
sites. As ESLs were collected, eggs were counted and examined under a dissecting microscope to 181 assess parasitism.

Egg sentinel cups (ESCs) were also tested for O. agrili recovery (Abell et al. 2015).

183 These were similar to the devices used to release $O$. agrili, however, the inner cups were

184 provisioned with 20 fresh ( $<5$ days old), non-parasitized EAB eggs on small pieces of filter paper

185 overnighted from the Brighton rearing facility and deployed the following day to serve as

186 potential hosts for actively seeking O. agrili females. One ESCs were attached to each of the 10

187 girdled trees at each site on the same date as ESLs were first deployed (28-Jun 2013) and used to

188 monitor for $O$. agrili parasitism weekly for four weeks ( $\mathrm{n}=80 \mathrm{ESC}$ samples). EAB eggs were

189 replaced with fresh eggs weekly and examined under a dissecting microscope two weeks after

190 being collected from the field (to allow for development of any O. agrili larvae) to visually

191 assess them for parasitism.

Larval sentinel logs (LSLs) used in S. agrili and T. planipennisi recovery were small

193 white ash logs ( $\sim 15 \mathrm{~cm}$ long, $\sim 5.5 \mathrm{~cm}$ diameter) provisioned with late instar EAB larvae (Abell

194 et al. 2015). We insured all logs used as LSLs had thin bark and a diameter of $<11.2 \mathrm{~cm}$ to

195 accommodate the short ovipositor of T. planipennisi (Abell et al. 2012). LSLs were infested

196 with EAB larvae by evenly distributing an average of six eggs on filter paper around the upper

197 portion of a $\log$ and securing them to the bark beneath a strip of Parafilm ${ }^{\circledR}$. Batches of $20 \operatorname{logs}$

198 were placed upright in covered plastic bins $(50 \times 35 \times 30 \mathrm{~cm})$ with ends submerged in $1-2 \mathrm{~cm}$ of

199 water to prevent desiccation and maintain humidity. LSLs were incubated at $\sim 31-33{ }^{\circ} \mathrm{C}$ for 5

200 weeks to allow larvae to develop to the $3^{\text {rd }}$ and $4^{\text {th }}$ instar. In total, only 55 of 80 LSLs were

201 successfully infested using this method and able to be used in the field. Prior to deployment, cut 202 ends of LSLs were coated with wood sealant (Anchorseal ${ }^{\circledR}$ ) to prevent desiccation. Each set of 
203 LSLs were exposed to parasitoids in the field for one week. LSLs were dissected and assessed

204 for healthy and parasitized EAB larvae two weeks after removal from the field (to allow

205 parasitoid larvae to mature to a size visible with the naked eye).

206 Twenty yellow pan traps (YPTs) were used for recovery of all parasitoid species

207 following methods previously developed and described (Bauer et al. 2016; USDA-

208 APHIS/ARS/FS 2016). YPTs were attached to all 10 girdled ash trees at the two field sites with a

209 shelf bracket and filled with a $20 \%$ clear propylene glycol solution to act as a preservative and

210 two drops of clear non-scented dish detergent to disrupt surface tension. Samples were collected

211 by pouring the contents of YPTs through a mesh paint strainer (mesh size $=226 \mu \mathrm{m})$ and

212 examined under a dissecting microscope for the presence of adult parasitoids. YPTs were

213 sampled weekly during the same four week period as all other trap types while parasitoids were

214 being actively released for comparison to other methods ( $\mathrm{n}=80$ YPT samples). Additionally,

215 YPT monitoring continued through 19 Oct 2013, after the other non-reusable trap types were

216 exhausted. Some data are presented for this extended period, however, they were not considered

217 in the actual methods comparisons, and are provided for informational purposes only.

218 During the O. agrili recovery portion of this experiment (28-Jun through 19-Jul), all

219 girdled trees had both YPTs and ESCs and a subset also equipped had ESLs. Each was assigned

220 a position around the trunk of each girdled tree at $90^{\circ}$ intervals relative to each other and to the

221 release device. Positions of traps were systematically alternated in relation to the release device

222 to remove placement bias relative to the release devise. On trees with only ESCs and YPTs, a

223 similar placement was used, just without the ESL trap type present. All traps were hung at a

224 height of $1.5 \mathrm{~m}$. 
226 through 13-Sept), empty $O$. agrili release devices were left in place for reference and a single

227 LSL and YPT was attached to each girdled tree, occupying positions as described above relative 228 to one another on each tree. Again, only 55 LSLs were produced and so a subset of trees had 229 only YPTs in some weeks. All traps were hung at a height of $1.5 \mathrm{~m}$.

230 Once all parasitoid recovery was completed, all 10 girdled trees from each site were 231 felled and cut into $1 \mathrm{~m}$ bolts and transported to cold storage. A $600 \mathrm{~cm}^{2}$ cylindrical section of 232 bark, centered on the midpoint of each bolt, was examined under a boom-mounted dissecting 233 microscope and assessed for egg-parasitism by $O$. agrili. Egg parasitism was determined based

234 on the presence of round emergence holes through the dorsal surface or sides of melanized EAB 235 eggs or presence of either wasp pupae or non-emerged adults within (Abell et al. 2014).

236 Following assessment of egg parasitism, the logs were debarked using a drawknife to assess

237 EAB larvae for parasitism by S. agrili, T. planipennisi, or other possible native parasitoids.

\subsubsection{Statistical analyses}

240 Regression analysis was used to determine the relationship between mean egg densities

241 on girdled trees and both total number of parasitized eggs and percentage egg parasitism. Mean

242 egg densities on girdled trees were estimated using the total number of EAB eggs observed

243 divided by the total surface area inspected. Percentage egg parasitism was calculated using $100 \mathrm{x}$

244 total number parasitized EAB eggs per tree/total number EAB eggs recovered per tree. A Mann-

245 Whitney U test was used for comparisons between both ESLs and LSLs with tree peeling due to

246 non-normally distributed egg and larval density data. A Chi-squared test of independence was

247 used to determine if there was association between positive/negative YPTs and presence/absence 
248 of parasitoids in corresponding girdled trees and Cramér's phi was calculated to determine the 249 degree of association. YPTs were considered positive based on recovery of one or more adult 250 parasitoids. Presence of $O$. agrili on girdled trees was defined as the presence of one or more 251 parasitized EAB egg(s). Presence of $S$. agrili and T. planipennisi in girdled trees was defined as

252 the presence of developing parasitoid larvae on one or more EAB larva(e). Fisher's exact test

253 was used whenever one or more Chi-square expected values were calculated to be less than five 254 for a given combination of positive/negative and presence/absence. All statistical analyses were 255 performed using Minitab 16. A summary table of all treatments is provided (Table 1).

$257 \quad 2.2$ Effective Range of Yellow Pan Traps

$258 \quad 2.2 .1$ Release Site

259 Parasitoids were released at eight points during summer 2014 within the Reed Road Bird 260 Refuge (53 ha) located in Monroe Co. near Chili, NY. Much of the Reed Road Bird Refuge is a 261 forested wetland with hydric soils supporting facultative wetland plant species, including a 262 canopy with a large green ash (Fraxinus pennsylvanica M.) and black ash (Fraxinus nigra M.)

263 component. The Reed Road Bird Refuge exhibits advanced signs of EAB infestation, including

264 most large dominant and codominant (mostly green ash) trees already killed by EAB and 265 evidence of extensive woodpecker foraging from several years previous.

\section{2.2.2 Parasitoid Release Points}

268 Eight parasitoid release points were selected in areas of the refuge with adequate numbers of 269 living ash trees allowing the most accurate deployment of yellow pan traps (YPTs) in rings at 270 two distances, $10 \mathrm{~m}$ and $20 \mathrm{~m}$. Central trees used for parasitoid release were chosen so there 
271 were 8 ash trees $\sim 10 \mathrm{~m}$ away and 16 ash trees $\sim 20 \mathrm{~m}$ away arranged in an approximate ring

272 pattern. These $10 \mathrm{~m}$ and $20 \mathrm{~m}$ rings were each replicated 4 times each for a total of 96 YPTs $(\mathrm{n}=$

$273 \quad 32$ at $10 \mathrm{~m}$ and $\mathrm{n}=64$ at $20 \mathrm{~m})$.

274 Distances from each release tree to trees intended for YPT deployment in a given ring were

275 measured, and the 8 or 16 ash trees closest to the desired distance of $10 \mathrm{~m}$ or $20 \mathrm{~m}$ were selected

276 to serve as YPT trees. YPTs were mounted on selected ash trees using a standard shelf bracket

$277(15 \times 20 \mathrm{~cm})$. Release trees were also affixed with a single YPT, placed in close proximity to $O$.

278 agrili release devices and T. planipennisi emergence bolts to document emergence and estimate

279 the beginning and end of parasitoid emergence periods for each species throughout the

280 experiment. Mean distance between release trees and YPTs for each ring and mean distance

281 between adjacent YPTs in the same ring are provided in Table 2.

282 YPTs were attached to either black ash $(n=34)$ or green ash $(n=62)$. All trees selected as

283 YPT trees were still alive at the beginning of the experiment and with the exception of several

284 small trees $(\sim 4.0-6.0 \mathrm{~cm} \mathrm{DBH})$, all were expected to harbor suitable EAB larval hosts given the

285 level of the infestation within the stand.

286

287 2.2.3 Parasitoid Releases and Recoveries

288 Oobius agrili and T. were released using protocols described in section 2.1.2. Release

289 devices contained 90 O. agrili-parasitized EAB eggs and were placed on the release tree at the

290 center of each of the four YPT rings on 2-Jul 2014 once 849 growing degree days (GDD, base

291 temperature $10^{\circ} \mathrm{C}$ ) had accumulated. Tetrastichus planipennisi were in 20 emergence bolts, four

292 of which were split in half vertically in order to evenly distribute bolts between all four release

293 points. All surfaces not covered in bark, including ends and exposed wood of bolts which were 
294 split, were coated in Anchorseal ${ }^{\circledR}$ green wood sealant to prevent desiccation. Two and one half 295 emergence bolts were placed on the release tree at the center of each YPT ring on 20-Aug 2014

296 once 1,667 GDD-B10C had accumulated. An estimated 575 T. planipennisi were expected to

297 emerge at each release point. YPTs were sampled once per week for five weeks following initial 298 deployment of emergence bolts on release trees in the field.

299 Both $O$. agrili release devices and T. planipennisi emergence bolts were left in the field for 300 one week after the end of each portion of the experiment to insure that any remaining parasitoids

301 were allowed additional time to emerge. Once removed from the field, parasitized EAB eggs

302 were examined under a dissecting microscope and $O$. agrili exit holes counted to quantify

303 emergence. The outer bark of T. planipennisi emergence bolts was removed and the number of

304 parasitized EAB larvae with T. planipennisi exit holes in the overlying bark was counted.

305 Because almost all broods had successfully emerged, this number was multiplied by the mean

306 number of T. planipennisi usually produced from a single EAB larva in a laboratory setting

307 (mean $=35$, Liu and Bauer 2007). Spathius agrili was not released in this experiment due to

308 limited availability.

\section{$310 \quad$ 2.3.4 Fixed-area Plots}

311 Tree data were collected from circular $200 \mathrm{~m}^{2}$ fixed-area plots with the parasitoid release tree 312 within each replicate serving as plot center. Species and DBH for all stems $\geq 5.0 \mathrm{~cm} \mathrm{DBH}$ were 313 recorded. From this, stem frequency, stem density, mean DBH, and basal area for black and 314 green ash was calculated. 
317 Emergence data for $O$. agrili and T. planipennisi were analyzed using one-way ANOVA to

318 determine differences in mean emergence of each species at the release point in each replicate. A

319 two-proportions test was performed to determine whether differences existed between the

320 proportion of parasitoid recoveries from YPTs on either black ash or green ash trees relative to

321 the number of each tree species with YPTs attached. Poisson regression analysis was performed

322 using count data to determine if there was a significant relationship between parasitoid recovery

323 and ash stem frequency or ash basal area surrounding release points. Analyses were performed

324 using Minitab 17 or R version 3.3.2.

325

326 3. Results

327 3.1 Comparison of Recovery Methods

328 3.1.1 Parasitoid Emergence

329 Adult $O$. agrili emerged from 1,849 of 2,490 (74.3\%) parasitized EAB eggs in the 20 release 330 devices deployed on the girdled trees at the two field sites. Adult S. agrili emerged from 1,122 of $3313,310(33.9 \%)$ cocoons counted during dissection of 397 parasitized EAB larvae in the 80 332 emergence logs, as evidenced by visible exit holes found in cocoon remnants. An estimated 3336,848 T. planipennisi successfully emerged from 214 of $285(75.1 \%)$ parasitized EAB larvae in 334 the 80 emergence logs based on presence of emergence holes through the bark overlying larval 335 galleries.

\subsubsection{Parasitoid Recovery}

338 Overall, girdled trap trees had the highest percentage of parasitoid recoveries compared to all 339 other methods (Table 3). YPTs followed with overall percentages of positive traps ranging from 
341 unsuccessful on all counts, as were LSLs for T. planipennisi. LSLs were somewhat successful

342 for S. agrili at $14.5 \%$, however, this was not significantly different that YPTs at $11.3 \%$ (Fisher's

343 exact test, $P=0.799)$. Capture details are provided below for each species.

344 Methods using fresh EAB eggs were unsuccessful in attracting O. agrili, as no parasitism of

345 EAB eggs was observed within ESCs or on ESLs at either site. EAB eggs on ESLs placed in the

346 field had a mean density of $338 \pm 51(\mathrm{SE}) \mathrm{eggs} / \mathrm{m}^{2}\left(\mathrm{n}=16\right.$, range $\left.116-811 \mathrm{eggs} / \mathrm{m}^{2}\right)$. During

347 sampling for naturally occurring EAB eggs on girdled trees $(\mathrm{n}=20), 10.4 \mathrm{~m}^{2}(21.1 \%)$ of total

348 bark surface area was inspected and EAB eggs were present at a mean density of $88 \pm 19$

$349 \mathrm{eggs} / \mathrm{m}^{2}\left(\mathrm{n}=20\right.$, range $\left.9-307 \mathrm{eggs} / \mathrm{m}^{2}\right)$. Although egg density was significantly lower on girdled

350 trees than on ESLs (Mann-Whitney U test, $P=<0.001$ ), successful egg parasitism was observed

351 on 12 of 20 girdled trees and $O$. agrili successfully parasitized 110 of 865 (12.7\%) recovered

352 EAB eggs. There was a significant positive linear relationship between the number of parasitized 353 eggs recovered and EAB egg density on girdled trees $\left(F_{1,18}=11.9, P=0.003, R^{2}\right.$ Adj $=36.4 \%$, Fig. 354 1).

355 Eight of 20 YPTs successfully captured a combined total of 28 of 1,849 (1.5\%) adult $O$.

356 agrili during the 4 wk recovery period and seven additional adult $O$. agrili during the remainder

357 of the season (2-Aug through 19-Oct). Successful recovery of adult $O$. agrili in YPTs was

358 associated with presence of $O$. agrili egg parasitism on the corresponding girdled tree to which a

359 given YPT was attached $\left(\chi^{2}=9.826, d f=1, \mathrm{n}=80, P=0.002\right)$. The effect size of this association

360 was moderate $\left(\varphi_{c}=0.35\right)$.

361 Tetrastichus planipennisi were recovered from YPTs and girdled trees, but not from LSLs.

362 There were 123 suitable EAB larvae $\left(4^{\text {th }}\right.$ instar and J-larvae at time of peeling) counted during 
363 dissection of LSLs, but none were parasitized. Parasitism was observed in 15 of 20 girdled trees

364 with 119 of 2,680 (4.4\%) suitable host larvae successfully parasitized by T. planipennisi. YPTs

365 successfully recovered 35 of an estimated 6,848 (0.51\%) adult $T$. planipennisi to emerge during

366 the designated $4 \mathrm{wk}$ recovery period immediately following release. An additional 83 adult $T$.

367 planipennisi were recovered during the five weeks following the designated recovery period

368 (once other traps had been removed) to quantify how many would be caught over a longer

369 period. There was no association between YPT recovery and presence or absence of $T$.

370 planipennisi within-tree larval parasitism due to frequent recovery in YPTs on trees with no

371 parasitized EAB larvae $\left(\chi^{2}=0.096, d f=1, \mathrm{n}=80, P=0.76\right)$. A significantly higher proportion of

372 females than male $T$. planipennisi were recovered in YPTs ( 88 vs $30, Z=-8.67, P=<0.001$ ).

373 Spathius agrili parasitized 8 of $123(6.5 \%)$ larvae in LSLs and parasitoid larvae were

374 recovered from 8 of 55 (14.5\%) LSLs containing EAB larva(e) suitable for parasitism. LSL

375 larval densities averaged $86.3 \pm 6.3$ larvae $/ \mathrm{m}^{2}$ and ranged from 38.6 to 231.7 larvae $/ \mathrm{m}^{2}$. Spathius

376 agrili larvae were recovered from 7 of 20 (35\%) girdled trees and parasitized 10 of 2,680 (0.37

$377 \%$ ) suitable EAB host larvae. Girdled tree larval densities were significantly lower than LSL

378 larval densities, averaging $50.4 \pm 9.8$ larvae $/ \mathrm{m}^{2}$ and ranging from 4.7 to 159.0 larvae $/ \mathrm{m}^{2}$ (Mann-

379 Whitney $\mathrm{U}$ test, $P=<0.001$ ). No significant difference existed between the overall proportion of

380 positive LSLs and positive girdled trees (Fisher's exact test, $P=0.179$ ). Successful recovery of $S$.

381 agrili parasitized EAB larvae in an LSL was not associated with presence or absence of larval

382 parasitism in the corresponding girdled tree to which the LSL was attached (Fisher's exact test, $P$ $383=0.416)$.

384 YPTs recovered $18(1.7 \%)$ of 1,122 S. agrili that successfully emerged over the entire

385 season. No significant difference existed between overall proportion of positive YPTs and LSLs 
386 (Fisher's exact test, $P=0.799$ ). Successful recovery of adult $S$. agrili from YPTs was not

387 associated with recovery of EAB larvae parasitized by S. agrili in corresponding LSLs (Fisher's

388 exact test, $P=0.082$ ). Successful recovery of adult $S$. agrili in YPTs was associated with

389 presence of $S$. agrili larval parasitism in the corresponding girdled tree to which a given YPT

390 was attached (Fisher's exact test, $P=0.048)$. The effect size of this association was low $\left(\varphi_{c}=\right.$

$3910.24)$.

392

393 3.2 Effective Range of Yellow Pan Traps

394 3.2.1 Parasitoid Emergence

395 A total of 3,008 (83.6\%) O. agrili adults emerged from parasitized EAB eggs placed at all 396 release points, with a mean emergence of $376 \pm 24$ (SE) individuals at each release point. With 397 the exception of one replicate, where two release devices were destroyed by wildlife, there were 398 no significant differences in total emergence among replicates.

399 An estimated total of 1,155 T. planipennisi adults emerged from all emergence bolts so that $400144 \pm 19$ (SE) emerged at each release point. Upon dissection of emergence bolts, exit holes 401 created by adult parasitoids confirmed T. planipennisi had successfully emerged from 33

402 parasitized EAB larvae. No significant differences in mean emergence for T. planipennisi were 403 observed among replicates.

404

\subsubsection{Parasitoid recovery}

406 Fifteen O. agrili were recovered in YPTs, all of which were associated with the four $20 \mathrm{~m}$ 407 release plots (Table 4). Eleven O. agrili were caught in release tree YPTs and four in ring 408 perimeter YPTs $20 \mathrm{~m}$ away. The first recovery of a single O. agrili occurred on 16-Jul 2014 from 
409 a release tree YPT. Ten $O$. agrili were recovered from two release tree YPTs the following week 410 on 23-Jul 2014, nine of which came from just one of these YPTs. This same week, four O. agrili 411 were recovered from ring perimeter YPTs in three of the four $20 \mathrm{~m}$ replicates. Two of these 412 individuals were recovered from separate, non-adjacent ring perimeter YPTs in the same $20 \mathrm{~m}$ 413 replicate. No additional O. agrili were recovered in release tree YPTs or $20 \mathrm{~m}$ ring perimeter 414 YPTs after 23-Jul 2014. There were no $O$. agrili recovered from release tree or ring perimeter 415 YPTs in any of the $10 \mathrm{~m}$ replicates during the entire experiment.

416 A total of 33 T. planipennisi were recovered in YPTs, 22 of which were caught in YPTs on 417 release trees, 4 in YPTs on trees $10 \mathrm{~m}$ from release points, and 7 in YPTs $20 \mathrm{~m}$ away (Table 4). 418 The first recovery of $T$. planipennisi individuals occurred on 3-Sept 2014, when one was 419 recovered from a release tree YPT and one was recovered from each of two ring perimeter YPTs 420 in separate $20 \mathrm{~m}$ replicates. YPTs then recovered $T$. planipennisi for an additional three 421 consecutive weeks. During the week of 10-Sept 2014, 12 T. planipennisi were recovered from 422 release tree YPTs in two $10 \mathrm{~m}$ replicates and all four $20 \mathrm{~m}$ replicates, a single individual was 423 recovered from one $10 \mathrm{~m}$ replicate, and three individuals were recovered from three separate 424 traps in two separate $20 \mathrm{~m}$ replicates. In the replicate that recovered two individuals, YPTs were 425 adjacent to one another. During the week of 17-Sept 2014, nine T. planipennisi were recovered 426 from release tree YPTs in two separate $20 \mathrm{~m}$ replicates, two individuals were recovered from 427 ring perimeter YPTs in two separate $10 \mathrm{~m}$ replicates, and two individuals were recovered from 428 ring perimeter YPTs in two separate $20 \mathrm{~m}$ replicates. During the week of 23-Sept 2014, one $T$. 429 planipennisi was recovered from a ring perimeter YPT in a $10 \mathrm{~m}$ replicate.

430 Oobius agrili was recovered from one ring perimeter YPT on green ash and three ring 431 perimeter YPTs on black ash; however, there was no significant difference between the 
432 proportions of positive YPTs to total number of YPTs on each ash species (Fisher's exact test: $P$

$433=0.126)$. Similarly, $T$. planipennisi was recovered from three YPTs placed on black ash and

434 eight YPTs placed on green ash and again no significant difference between proportions was

435 observed (Fisher's exact test: $P=0.366$ )

436 Regression analysis showed O. agrili recovery was not correlated with ash stem density

$437\left(F_{1,6}=0.3, P=0.872\right)$, however, there was a significant quadratic relationship between $T$.

438 planipennisi recovery and ash stem density $\left(\mathrm{F} 1,6=7.92, \mathrm{P}=0.037, \mathrm{R}_{\text {Adj }}^{2}=69.7 \%\right)$. Poisson

439 regression indicated a non-significant effect of YPT distance on total parasitoid recovery $(\beta=$

$440 \quad 0.20 ; 95 \%$ CI: $-1.11,1.31 ; P=0.77)$, but yielded a significant relationship between total

441 parasitoid recovery (O. agrili and T. planipennisi combined) and total ash basal area within each

442 replicate $(\beta=2.39 ; 95 \% \mathrm{CI}: 1.18,4.87 ; P=0.016)$. This indicates total parasitoid capture

443 increased by 2.39 times for every square meter increase in ash basal area (Fig. 2).

$444 \quad$ Finally, general direction from release trees to all YPTs where parasitoids were recovered

445 was recorded, demonstrating the greatest numbers of parasitoids were recovered to the north and

446 northeast of release points. A wind rose diagram was adapted using count data to create a visual

447 representation of general patterns of parasitoid dispersal direction (Fig. 3).

449 4. Discussion

$450 \quad$ 4.1 Comparison of Recovery Methods

451 Although the presence of $O$. agrili was confirmed by recovery of parasitized EAB eggs on 452 girdled trees and adult wasps were recovered in YPTs at both release sites in 2013, ESCs and 453 ESLs were unsuccessful in documenting egg parasitism. Unfortunately, ESL sample size was 454 much smaller than desired due to difficulties encountered in making ESLs, i.e. low survivorship 
455 of adult EAB in captivity resulted in few sexually mature females for egg production and

456 oviposition. Despite higher mean EAB egg densities on ESLs than girdled trees, even the

457 maximum egg density achieved on an ESL used in this study $\left(811 \mathrm{eggs} / \mathrm{m}^{2}\right)$ was lower than mean

458 densities $\left(\sim 1,265\right.$ eggs $\left./ \mathrm{m}^{2}\right)$ on ESLs used by Duan et al. (2012) during 2009 and 2010. This may

459 mean there were insufficient egg densities necessary to attract or entice $O$. agrili to remain in

460 close proximity long enough for parasitism to occur. It is also possible EAB eggs presented in

461 ESCs and on ESLs were not preferable for $O$. agrili parasitism at the time of exposure, as $O$.

462 agrili has been shown to prefer younger eggs (Duan et al. 2014). To accommodate this, all EAB

463 eggs used were $<5$ days old when placed in the field and therefore $<12$ days old at time of

464 collection. However, ambient temperatures $>24^{\circ} \mathrm{C}$ during Jun 2013 and potentially higher

465 temperatures within ESCs exposed to direct sunlight may have reduced egg preferability to an

466 even shorter time period.

467 Additionally, O. agrili used by Duan et al. (2012) were released as pre-nourished adults.

468 Laboratory reared $O$. agrili have been shown to die within $24 \mathrm{hr}$ of emergence unless provided

469 with honey (L Bauer, unpublished data). Though there was emergence of large numbers of adult

470 O. agrili, it is possible they failed to locate honey provided within release cups and dispersed

471 away from ESLs in search of alternative food resources on the main trunk or other locations

472 before parasitism could occur.

473 Though relatively little is known about the flight capabilities of $O$. agrili, association

474 between recoveries of adults in YPTs and presence of parasitized eggs on girdled trees suggest a

475 close relationship between $O$. agrili and its tree of origin. There was only one instance wherein

476 o. agrili was recovered in a YPT on a girdled tree from which no parasitized EAB eggs were

477 recovered during sampling. YPTs were less reliable predictors of S. agrili or T. planipennisi 
478 parasitism within girdled trees, as each species was recovered on multiple occasions in YPTs on

479 girdled trees containing no parasitoid larvae. This suggests the larger S. agrili and T. planipennisi

480 likely disperse away from their natal tree more often, highlighting the potential role that

481 proximity to abundant host-bearing ash trees may play in efficacy of YPTs for different

482 parasitoid species.

483 Both LSLs and YPTs proved to be successful methods of S. agrili recovery and were no less

484 effective at detecting presence of S. agrili than destructive sampling of entire girdled trees.

485 Because only one EAB larvae was parasitized in each positive LSL, further correlation could not 486 be established between parasitism rates in LSLs and girdled trees. Although YPTs did not 487 provide information on parasitism rates in this study, our results indicate YPTs are as effective as 488 LSLs for simple detection of S. agrili. Given these results, it is also promising that YPTs might 489 be effective for monitoring Spathius galinae Belokobylskij \& Strazanac (Hymenoptera:

490 Braconidae), another recently-described and approved biological control agent of EAB now 491 being released in a limited number of states (Federal Register 2015).

492 Unlike S. agrili, T. planipennisi was not recovered using LSLs and was only recovered in 493 YPTs. Although it was not likely that $T$. planipennisi females were physically prevented from 494 ovipositing through thin bark of small LSLs, i.e. bark did not exceed their ovipositor length 495 (Abell et al. 2012), no T. planipennisi-parasitized larvae were found. Previous studies using 496 similar bolts have demonstrated successful parasitism of LSLs by T. planipennisi in MI (Bauer et 497 al. 2011); however, LSLs used to recover T. planipennisi during those trials contained late-instar 498 larvae manually inserted into small cavities excavated beneath bark flaps, which is different in 499 that ours contained larvae hatched from eggs placed on logs. Bauer et al. (2011) also indicated 500 LSLs with manually inserted larvae were preferred by $T$. planipennisi to those with naturally 
501 developing larvae, indicating either a change in host apparency or that females were able to 502 access larvae more easily through the bark flaps when that method was used.

503 When hosts are limited, female S. agrili have also been shown to exclude T. planipennisi

504 females in lab and field trials by means of more efficient host location and unwillingness of $T$. 505 planipennisi to utilize larvae immobilized by S. agrili parasitism (Ulyshen et al. 2011). This is

506 presumably due to reliance by both species on host vibrations as a way to locate hosts, in which

507 case larvae already paralyzed by S. agrili during oviposition would be undetectable to searching

508 T. planipennisi females (Ulyshen et al. 2011). Regardless, in this study, S. agrili did not

509 parasitize larvae in LSLs to a degree that $T$. planipennisi would have been excluded.

510 YPTs were the only method besides whole tree dissection to recover all three species of

511 released parasitoids. Unlike female $O$. agrili, which can engage in host feeding on fluids from

512 exposed EAB eggs during oviposition (Bauer, personal observation), parasitoids of concealed

513 larvae such as S. agrili and T. planipennisi are presumed to seek floral nectar or insect honeydew

514 for nourishment. Colored pan traps, intended to mimic flower or foliage reflectance, have often

515 been used to collect insects and yellow is often selected for its ability to attract parasitic

516 hymenopterans (Noyes 1989, Pucci 2008). In addition to yellow, preferences for white and green

517 landing surfaces have been demonstrated for S. agrili (Cooperband 2013). Although color

518 preferences for $O$. agrili or T. planipennisi have not yet been investigated, YPTs recovered

519 similar overall percentages of all three species over the entire season.

520 At least one T. planipennisi was recovered in YPTs each week from 23-Aug 2013 until

521 sampling concluded on 19-Oct 2013. Under laboratory settings, it takes T. planipennisi roughly 4

522 weeks to complete a generation and adult males and females have a median lifespan of 5 and 6

523 weeks, respectively (Duan et al. 2011). Tetrastichus planipennisi females were recovered a full 
524 six weeks after the final set of emergence logs were placed in the field. It is possible these were

525 long-lived individuals; however, given the reduced life expectancy of adult insects in the field,

526 we believe YPTs were able to recover some progeny of parasitoids released early in our

527 experiment.

528 Although a greater number of T. planipennisi females than males were caught using YPTs,

529 the ratio of females to males caught is consistent with the expected 3:1 female to male sex ratio

530 observed in laboratory populations (Duan et al. 2011) and so does not indicate differential

531 attraction to YPTs between sexes.

532

533 4.2 Effective Range of Yellow Pan Traps

534 Recoveries of $O$. agrili adults in YPTs attached to release trees $(\mathrm{n}=8)$ was not surprising, 535 however, capturing them in traps $20 \mathrm{~m}$ from the point of release $(\mathrm{n}=64)$ was unexpected. It is

536 unknown why none were captured in trees $\sim 10 \mathrm{~m}$ from dispersal points, however, that they were

537 recovered in traps $20 \mathrm{~m}$ away revealed dispersal capabilities greater than previously thought.

538 Though perhaps able to fly directly to ash trees, it is more likely, given the minute size of $O$.

539 agrili, that adults were able to locate ash trees during passive, wind-aided dispersal. Because

540 EAB females oviposit on the bark surface, it is doubtful any induced host tree response occurs

541 until neonate EAB larvae entering the tree begin to feed. Insect eggs typically lack long-range

542 chemical cues (Fatouros et al. 2008) and therefore cues used by egg parasitoids likely originate

543 with the host plant itself, with adult EAB, or a combination of these factors. If $O$. agrili is a

544 passive disperser, cues used in host egg location are likely responsible for informing $O$. agrili

545 when to remain in a potentially host rich environment, rather than attracting $O$. agrili to such an

546 environment from a distance. 
Similar to observations made while releasing adults from cups, T. planipennisi emerging

548 from emergence bolts appears to have also dispersed rapidly, as evidenced by recoveries of $T$.

549 planipennisi at a distance of $20 \mathrm{~m}$ during the first week that individuals also appeared in YPTs

550 on release trees. Tetrastichus planipennisi appeared to remain active within release sites

551 throughout the entire sampling period, with recovery of at least one individual at both distances

552 during all four weeks.

553 With the exception of one release site where green ash was absent in the fixed-area plot,

554 relative abundance of black ash and green ash were similar at all release points. Although black

555 ash is considered highly susceptible to EAB (Rebek et al. 2008), differences in overall health of

556 small black ash and green ash in this study were not apparent. If parasitoid recovery was

557 influenced by attraction to ash trees rather than YPTs, neither O. agrili nor T. planipennisi

558 appeared to demonstrate preference for ash species and similar numbers of individuals were

559 recovered from YPTs on green ash and black ash for both parasitoid species.

560 Other characteristics of ash trees surrounding release points in fixed-area plots that might

561 affect recovery and dispersal, such as ash stem density and ash basal area, were predictors of

562 parasitoid recovery in YPTs within the same replicate. Greater frequencies of parasitoid

563 recoveries in replicates with higher ash stem density and ash basal area most likely reflects

564 increased availability of high-quality patches of suitable hosts. Parasitoids frequently

565 encountering high-quality patches of hosts might have reduced incentive to disperse and may

566 have remained within the confines of YPT rings for a longer period of time, thus increasing

567 exposure time to YPTs during weekly sampling periods. Conversely, YPT rings with lower ash

568 stem frequency and ash basal area where parasitoid recovery was low or absent may reflect 
569 parasitoids greater tendency to disperse in search of areas of higher ash density, presumably

570 perceived as higher quality host environment by seeking females.

571 Although cases of dispersal were observed in all cardinal directions, the majority of $O$. agrili

572 and T. planipennisi were recovered in YPTs placed on trees to the north or northeast of release

573 points. All YPTs were oriented towards the release point when attached to trees, meaning these

574 YPTs were placed on the southern or southwestern faces of ash trees and would likely have

575 received greater amounts of direct sunlight. This could have resulted in a change in apparency

576 and increased attractiveness of YPTs to dispersing parasitoids. In addition, EAB females are

577 shown to preferentially oviposit on the side of ash trees with greatest exposure to sunlight

578 (Timms et al. 2006). Higher frequency of parasitoid recovery from YPTs oriented in these

579 directions could illustrate a foraging response to increased availability of EAB egg and larval 580 hosts.

\section{Conclusions}

583 Although field research involving parasitoids remains difficult to pursue due to inherent 584 challenges associated with observing populations of small and cryptic insects, results of this

585 study indicate YPTs are comparable to several other methods for recovery and require a fraction 586 of the overall effort associated with other more elaborate and sometimes less predictable 587 methods. Compared to other traps, YPTs can be more easily mass-produced, can be produced at 588 a moment's notice, and can be sampled with a high degree of flexibility depending on the user's 589 needs. Most importantly, YPTs are unique in their ability to recover all three species of EAB 590 parasitoids being released during this experiment. Moreover, we found that YPTs can recover $O$. 591 agrili and at greater distances than previously thought. 
593 EAB parasitoids are established and attacking EAB. Unfortunately, it was beyond the scope of

594 this study to conclusively document YPT recoveries as indicators of percent parasitism for all

595 parasitoid species and impacts they are exerting on EAB populations. For the purpose of

596 obtaining this information, there is still no better method than dissecting trees. When this is not

597 feasible however, YPTs are recommended as an efficient means of monitoring parasitoid

598 presence and dispersal, especially as states continue to release increasing numbers of parasitoids

599 and new states begin to participate in release programs.

600

601

602

603

604

605

606

607

608

Acknowledgements

609 We would like to thank the USDA EAB Parasitoid Rearing Facility in Brighton, MI for

610 producing and supplying all parasitoids used in this study. We also thank the Northern States

611 Research Cooperative for funding of this project, the Genesee Land Trust for allowing us use of

612 the Reed Road Bird Refuge, and the NYS Department of Environmental Conservation for

613 supplying us with a research vehicle. Finally, special thanks to Jonathan Lelito (USDA APHIS

614 PPQ) for expert advice on rearing parameters for EAB larvae used in larval sentinel logs, Tracy 
615 Ayer (USDA APHIS PPQ) for supplemental production of egg sentinel logs, and Nicholas

616 Piedmonte for assistance with statistical analyses.

\section{References}

618 Abell K.J., Duan J.J., Bauer L.S., Lelito J.P. and Van Driesche R. (2012). The effect of bark

619 thickness on host partitioning between Tetrastichus planipennisi (Hymenoptera: Eulophidae)

620 and Atanycolus spp. (Hymenoptera: Braconidae), two parasitoids of emerald ash borer

621 (Coleoptera: Buprestidae). Biological Control. 63(3): 320-325.

622 Abell K.J., Bauer L.S., Duan J.J., and Van Driesche R. (2014). Long-term monitoring of the

623 introduced emerald ash borer (Coleoptera: Buprestidae) egg parasitoid, Oobius agrili

624 (Hymenoptera: Encyrtidae), in Michigan USA and evaluation of a newly developed

625 monitoring technique. Biological Control. 79: 36-42.

626 Abell, K.J., Poland, T., Cossé, A., and Bauer, L.S. 2015. Trapping techniques for emerald ash

627 borer and its introduced parasitoids. In: Van Driesche, R.G. and Reardon, R.C. (eds).

628 Biology and control of emerald ash borer. USDA Forest Service, FHTET 2014-09, pp. 113-

$629 \quad 128$.

630 Bauer, L. S., H-P. Liu, D. L. Miller, and J. Gould. 2008. Developing a classical biological

631 control program for Agrilus planipennis (Coleoptera: Buprestidae), an invasive ash pest in North America. Newsletter of the Michigan Entomological Society 53: 38-39.

Bauer L.S., Gould J.R., Duan J.J., Hansen J.A., Cossé A.A., Miller D.L., Abell K.J., Van

635 Driesche R., Lelito J.P., and Poland T.M. (2011). Sampling methods for recovery of exotic 636 emerald ash borer parasitoids after environmental release. pp. 2-5. In: McManus, K. and K.

637 Gottschalk (eds.). Proceedings of the $22^{\text {nd }}$ USDA Interagency Research Forum on Invasive 

$\underline{\text { http://nrs.fs.fed.us/pubs/39810 }}$

Bauer, L.S., Duan J.J., and Gould J.R. 2014. Emerald ash borer (Agrilus planipennis Fairmaire)

641 (Coleoptera: Buprestidae). Ch. 17. Pp. 189-205. In The Use of Classical Biological Control

642 to Preserve Forests in North America. (Ed. R. Van Driesche and R. Reardon) United States

643 Department of Agriculture, Forest Service, FHTET-2013-02.

644 Bauer L.S., Duan J.J, Gould J.R., and Van Driesche R. (2015). Progress in the classical

645 biological control of Agrilus planipennis Fairmaire (Coleoptera: Buprestidae) in North

646 America. Canadian Entomologist. 147: 300-317.

647 Bauer L., Hansen J., and Gould J. 2016. Yellow Pan Traps: A Simple Method for Trapping

648 Parasitoids Released for Biological Control of the Emerald Ash Borer.

649 http://www.nrs.fs.fed.us/disturbance/invasive_species/eab/local-

650 resources/downloads/YPT_Method20141210.pdf

651 Belokobylskij S.A., Yurchenko G.I., Strazanac J.S., Zaldívar-Riverón A., and Mastro V. (2012).

652 A New Emerald Ash Borer (Coleoptera: Buprestidae) Parasitoid Species of Spathius Nees

653 (Hymenoptera: Braconidae: Doryctinae) From the Russian Far East and South Korea. Annals 654 of the Entomological Society of America. 105(2): 165-178.

655 Cooperband M.F., Hartness A., Lelito J.P., and Cossé A.A. (2013). Landing surface color 656 preferences of Spathius agrili (Hymenoptera: Braconidae), a parasitoid of emerald ash borer, 657 Agrilus planipennis (Coleoptera: Buprestidae). Journal of Insect Behavior. 26: 721-729.

658 Duan J.J., Oppel C.B., Ulyshen M.D., Bauer L.S. and Lelito J.P. (2011). Biology and life history 659 of Tetrastichus planipennisi (Hymenoptera: Eulophidae), a larval endoparasitoid of the 660 emerald ash borer (Coleoptera: Buprestidae). 94(4): 933-940. 
661 Duan J.J., Bauer L.S., Hansen J.A., Abell K.J. and Van Driesche R. (2012). An improved method 662 for monitoring parasitism and establishment of Oobius agrili (Hymenoptera: Encyrtidae), an 663 egg parasitoid introduced for the biological control of emerald ash borer (Coleoptera:

664 Buprestidae) in North America. Biological Control. 60(3): 255-261.

665 Duan J.J., Bauer L.S., Abell K.J., Ulyshen M.D., and Van Driesche R.G. (2015). Population 666 dynamics of an invasive forest insect and associated natural enemies in the aftermath of 667 invasion: Implications for biological control. Journal of Applied Ecology. 52: 1246-1254. 668 Cappaert D., McGullough D.G., Poland T.M. and Siegert N.W. (2005). Emerald ash borer in 669 North America: A research and regulatory challenge. American Entomologist. 51(3): 152$670 \quad 163$.

671 Fatouros N.E., Dicke M., Mumm R., Meiners T. and Hilker M. (2008). Foraging behavior of egg 672 parasitoids and exploiting chemical information. Behavioral Ecology. 19(3): 677-689.

673 Federal Register (2007). Availability of an environmental assessment for the proposed release of 674 three parasitoids for the biological control of the emerald ash borer (Agrilus planipennis) in 675 the continental United States. Federal Register. 72: 28947-28948. [Docket No. APHIS676 2007-006].

677 Federal Register (2015). Field release of the parasitoid Spathius galinae for the biological control 678 of the emerald ash borer (Agrilus planipennis) in the continental United States. Federal 679 Register. 80: 7827 [Docket No. APHIS-2014-0094].

680 Gould J.R., Bauer L.S. and Duan J.J. 2016. Emerald Ash Borer, Agrilus planipennis (Fairmaire), 681 Biological Control Release and Recovery Guidelines. USDA APHIS-FS-ARS, Riverdale, 682 Maryland. 
683 Herms D.A. and McCullough D.G. (2014). Emerald ash borer invasion of North America:

684 History, Biology, Ecology, Impacts, and Managements. Annual Review of Entomology. 59:

$685 \quad 13-30$.

686 Jennings D.E., Duan J.J., Larson K.M., Lelito J.P. and Shrewsbury P.M. (2014). Evaluating a

687 new method for monitoring the field establishment and parasitism of Oobius agrili

688 (Hymenoptera: Encyrtidae), an egg parasitoid of emerald ash borer (Coleoptera:

689 Buprestidae). Florida Entomologist 97: 1263-1265.

690 Liu H., Bauer L.S., Gao R., Zhao T., Petrice T.R. and Haack R.A. (2003). Exploratory survey for

691 the emerald ash borer, Agrilus planipennis (Coleoptera: Buprestidae), and its natural enemies

692 in China. Great Lakes Entomologist. 36(3, 4): 191-204.

693 Liu H. and Bauer L.S. (2007). Tetrastichus planipennisi (Hymenoptera: Eulophidae), a

694 gregarious larval endoparasitoid of emerald ash borer. Proceedings of the 2006 Emerald Ash

695 Borer Research and Technology Development Meeting. Cincinnati, OH. Oct. 29-Nov. 2,

696 2006. USDA Forest Service, Morgantown, WV. FHTET 2007-04.

697 MapBioControl. 2014. Agent release tracking and data management for federal, state, and

698 researchers releasing three biocontrol agents released against emerald ash borer.

$699 \quad$ http://www.mapbiocontrol.org

700 Minitab 16 Statistical Software (2010). [Computer software]. State College, PA: Minitab, Inc.

701 Minitab 17 Statistical Software (2014). [Computer software]. State College, PA: Minitab, Inc.

702 McCullough D.G., Poland T.M. and Cappaert D. (2009). Attraction of the emerald ash borer to

703 trees stressed by girdling, herbicide treatment, or wounding. Canadian Journal of Forest

$704 \quad$ Research. 39: 1331-1345. 
Noyes J.S. (1989). A study of five methods of sampling Hymenoptera (Insecta) in a tropical rainforest, with special reference to the Parasitica. Journal of Natural History. 23: 285-298.

Pucci T. (2008). A comparison of the parasitic wasps (Hymenoptera) at elevated versus ground yellow pan traps in a beech-maple forest. Journal of Hymenoptera Research. 17: 116-123.

R Core Team (2016). R; A language and environment for statistical computing. R Foundation for

$$
\text { Statistical Computing, Vienna, Austria. http://R-project.org }
$$

Rebek E.J., Herms D.A., and Smitley D.R. (2008). Interspecific variation in resistance to emerald ash borer (Coleoptera: Buprestidae) among North American and Asian ash (Fraxinus spp.). Environmental Entomology. 37(1): 242-246.

Timms L.L., Smith S.M. and de Groot P. (2006). Patterns in the within-tree distribution of the emerald ash borer Agrilus planipennis (Fairmaire) in young, green-ash plantations in south-

western Ontario, Canada. Agricultural and Forest Entomology. 8: 313-321.

Ulyshen M.D., Duan J.J. and Bauer L.S. (2011). Interactions between Spathius agrili (Hymenoptera: Braconidae) and Tetrastichus planipennisi (Hymenoptera: Eulophidae), larval parasitoids of Agrilus planipennis (Coleoptera: Buprestidae). Biological Control. 52: 188726 parasitoid from China of Agrilus planipennis: A new species of Spathius (Hymenoptera: 193.

USDA-APHIS/ARS/FS (2016). Emerald Ash Borer Biological Control Release and Recovery Guidelines. USDA-APHIS-ARS-FS. Riverdale, MD.

Vrdoljak S.M. and Samways M.J. (2012) Optimising coloured pan traps to survey flower visiting insects. Journal of Insect Conservation. 16: 345-354

Yang Z.Q., Strazanac J.S., Marsh P.M., Van Achterberg C. and Choi W.Y. (2005). First recorded Braconidae: Doryctinae). Annals of the Entomological Society of America. 98(5): 636-642. 
728 Yang Z.Q., Strazanac J.S., Yao Y.X. and Wang X.Y. (2006). A new species of emerald ash borer

729 parasitoid from China belonging to the genus Tetrastichus Haliday (Hymneoptera:

730 Eulophidae). Proceedings of the Entomological Society of Washington. 108(3): 550-558.

731 Zhang Y.Z., Huang D.W., Zhao T.H., Liu H.P. and Bauer L.S. (2005). Two new species of egg

732 parasitoids (Hymenoptera: Encyrtidae) of wood-boring beetle pests from China.

733 Phytoparasitica. 33(3) 253-260. 
Table 1. Number of each trap type used and EAB host egg and/or larvae availability for each trap type evaluated during recovery method comparison experiment. Note that YPTs are not provisioned with any form of EAB egg or larval host.

\begin{tabular}{|c|c|c|c|c|c|}
\hline RECOVERY METHOD & $\begin{array}{l}\text { TOTAL NUMBER } \\
\text { EVALUATED }\end{array}$ & $\begin{array}{l}\text { MIN HOSTS } \\
\text { AVAILABLE }\end{array}$ & $\begin{array}{l}\text { MAX HOSTS } \\
\text { AVAILABLE }\end{array}$ & $\begin{array}{l}\text { AVERAGE Hosts } \\
\text { AVAILABLE }\end{array}$ & $\begin{array}{c}\text { Total Hosts } \\
\text { AvAILABLE }\end{array}$ \\
\hline GIRDLED TREES & 20 & $\begin{array}{c}5 \text { EGGS, } \\
12 \text { LARVAE }\end{array}$ & $\begin{array}{c}129 \text { EGGS, } \\
407 \text { LARVAE }\end{array}$ & $\begin{array}{c}43 \text { EGGS, } \\
\text { 151 LARVAE }\end{array}$ & $\begin{array}{c}865 \text { EGGS, } \\
\text { 3019 LARVAE }\end{array}$ \\
\hline $\begin{array}{l}\text { EGG SENTINEL LOG } \\
\text { (ESL) }\end{array}$ & 16 & 3 EGGS & 21 EGGS & 8-9 EGGS & 140 EGGS \\
\hline $\begin{array}{l}\text { EGG SENTINEL CUP } \\
\text { (ESC) }\end{array}$ & 80 & 20 EGGS & 20 EGGS & 20 EGGS & 1600 EGGS \\
\hline $\begin{array}{l}\text { LARVAL SENTINEL LOG } \\
\text { (LSL) }\end{array}$ & 55 & 1 LARVA & 5 LARVAE & 2-3 LARVAE & 123 LARVAE \\
\hline $\begin{array}{l}\text { YELLOW PAN TRAPS } \\
\text { (YPT) }\end{array}$ & 80 & - & - & - & - \\
\hline
\end{tabular}


Table 2. Summary measurements of mean distance and range of distances between release trees and YPTs and between YPTs and adjacent YPTs for all replicates.

\begin{tabular}{|c|c|c|c|c|}
\hline REPLICATE & $\begin{array}{c}\text { MEAN DistANCE FROM } \\
\text { CENTER }(\mathrm{M}) \pm \mathrm{SE}\end{array}$ & RANGE (M) & $\begin{array}{c}\text { MEAN DiSTANCE FROM } \\
\text { ADJACENT YPTS (M) } \pm \text { SE }\end{array}$ & RANGE (M) \\
\hline $10 \mathrm{M}-1$ & $10.4 \pm 0.5$ & $8.3-12.7$ & $7.6 \pm 1.6$ & $2.9-16.8$ \\
\hline $10 \mathrm{M}-2$ & $10.2 \pm 0.7$ & $7.4-12.6$ & $7.4 \pm 0.7$ & $5.2-10.0$ \\
\hline $10 \mathrm{M}-3$ & $10.7 \pm 0.3$ & $9.7-12.2$ & $8.0 \pm 0.9$ & $5.2-13.2$ \\
\hline $10 \mathrm{M}-4$ & $10.3 \pm 0.3$ & $9.4-11.9$ & $7.7 \pm 0.8$ & $4.0-10.4$ \\
\hline $20 \mathrm{M}-1$ & $19.0 \pm 0.5$ & $15.2-21.6$ & $7.4 \pm 1.0$ & $2.8-17.0$ \\
\hline $20 \mathrm{M}-2$ & $19.4 \pm 0.2$ & $18.0-20.5$ & $7.5 \pm 1.2$ & $1.6-19.3$ \\
\hline $20 \mathrm{M}-3$ & $21.7 \pm 0.7$ & $16.6-25.6$ & $8.8 \pm 1.1$ & $2.0-15.3$ \\
\hline $20 \mathrm{M}-4$ & $20.2 \pm 0.3$ & $17.2-22.1$ & $7.8 \pm 1.0$ & $2.4-17.7$ \\
\hline
\end{tabular}


Table 3. Overall percentage of positive traps for each trap type by parasitoid species.

\begin{tabular}{|c|c|c|c|}
\hline $\begin{array}{c}\text { RECOVERY } \\
\text { METHOD }\end{array}$ & $\begin{array}{c}\text { POSITIVE FOR } \\
\text { O. AGRILI }\end{array}$ & $\begin{array}{c}\text { POSITIVE FOR } \\
\text { T. PLANIPENNISI }\end{array}$ & $\begin{array}{c}\text { POSITIVE FOR } \\
\text { S. AGRILI }\end{array}$ \\
\hline GIRDLED TREES & $60 \%$ & $75 \%$ & $35 \%$ \\
\hline $\begin{array}{c}\text { EGG SENTINEL } \\
\text { LOG (ESL) }\end{array}$ & 0 & NA & NA \\
\hline $\begin{array}{c}\text { EGG SENTINEL } \\
\text { CUP (ESC) }\end{array}$ & 0 & NA & $14.5 \%$ \\
\hline $\begin{array}{c}\text { LARVAL SENTINEL } \\
\text { LOG (LSL) }\end{array}$ & NA & $22.5 \%$ & $11.3 \%$ \\
\hline $\begin{array}{c}\text { YELLOW PAN } \\
\text { TRAPS (YPT) }\end{array}$ & $17.5 \%$ & 0 & \\
\hline
\end{tabular}


Table 4. Parasitoid recoveries from YPTs on release trees and ring YPTs at each distance on by sampling date.

\begin{tabular}{|c|c|c|c|c|c|c|c|c|c|c|}
\hline \multirow{2}{*}{ YPT LOCATION } & \multicolumn{5}{|c|}{ o. agrili RECOVERY } & \multicolumn{5}{|c|}{ T. planipennisi RECOVERY } \\
\hline & $7 / 9$ & $7 / 16$ & $7 / 23$ & $7 / 30$ & $8 / 6$ & $8 / 27$ & $9 / 3$ & $9 / 10$ & $9 / 17$ & $9 / 23$ \\
\hline 10 M RELEASE TREE & & & & & & & 1 & 2 & 6 & \\
\hline $10 \mathrm{M}$ RING & & & & & & & & 1 & 2 & 1 \\
\hline 20 M RELEASE TREE & & 1 & 10 & & & & & 10 & 3 & \\
\hline 20 M RING & & & 4 & & & & 2 & 3 & 2 & \\
\hline
\end{tabular}






Figure 1. Responses of $O$. agrili to varying EAB egg densities on girdled ash trees on which they were released $\left(F_{1,18}=11.9, P=0.003, R_{\text {Adj }}^{2}=36.4 \%\right)$. EAB egg density is given in the number of EAB eggs found on each $\mathrm{m}^{2}$ of bark. Dashed lines depict $95 \%$ confidence intervals. 


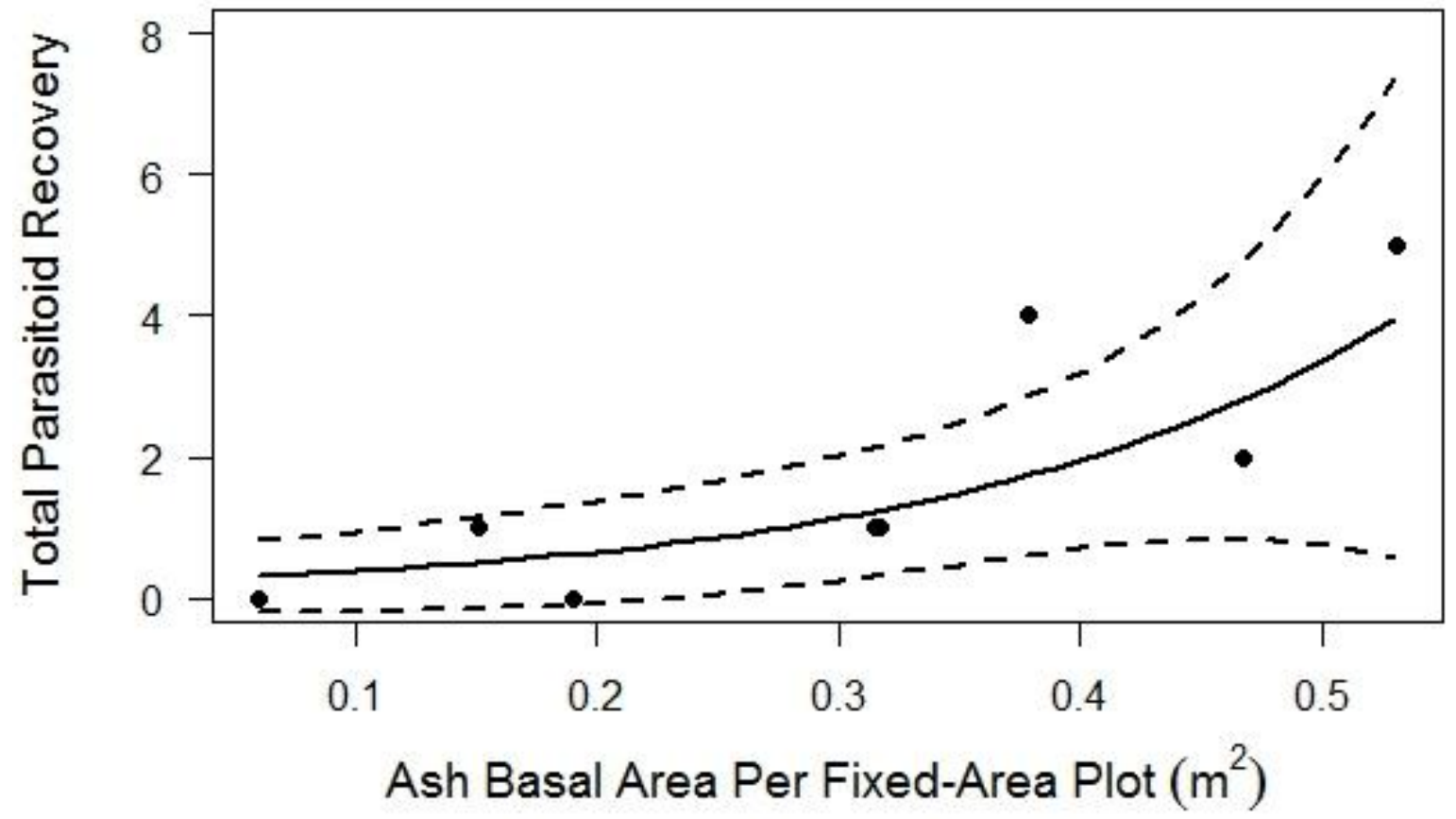

Figure 2. Rings of yellow pan traps (YPTs) surrounding fixed-area plots with higher ash basal area recovered more parasitoids overall than those with lower ash basal area $(\beta=2.39 ; 95 \%$ CI: $1.18,4.87 ; P=0.016)$. 


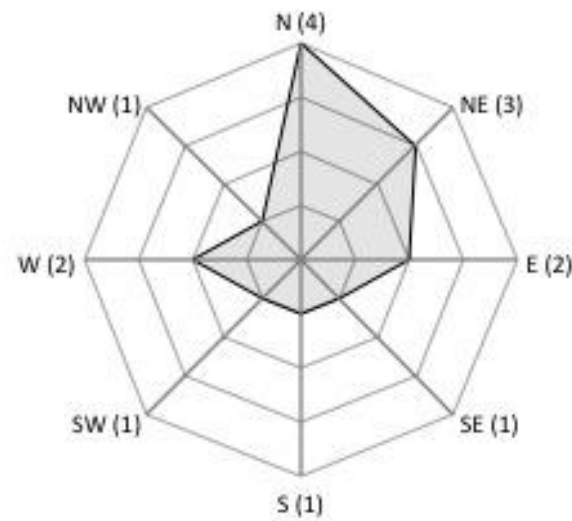

Figure 3. Combined recoveries of $O$. agrili and T. planipennisi and the general direction of dispersal from the release tree to YPTs with parasitoid recoveries. The number in parentheses accompanying each direction label indicates the total number of individual parasitoids recovered in that direction. 